\title{
Das Schematismuskapitel in der Kritik der reinen Vernunft.
}

Philologische Untersuchung.

Von Ernst Robert Curtius.

$\mathrm{Zu}$ den verrufensten Partien in der „Kritik der reinen Vernunft" gehört das Kapitel "von dem Schematismus der reinen

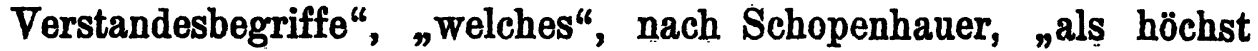
dunkel berühmt ist, weil kein Mensch je hat daraus klug werden. können“. ${ }^{1}$ ) „Seine Stellung," sagt der Verfasser der letzten Arbeit über den Gegenstand, „ist nicht ohne weiteres erkennbar, seine Darstellung unleugbar schwierig, bisweilen dunkel, die ersten Eindrücke, die man von seinem Sinne empfängt, so befremdend, dass es jederzeit Verwunderung erregte und einem zeitgenössischen Gegner Kants wie ein ,metaphysischer Roman" vorkam" (nach dem Bericht von Jak. Sieg. Beck: „einzigmöglicher Standpunkt“, S. 56). [Dr. H. Levy, Kants Lehre von dem Schematismus der reinen Verstandesbegriffe, I. 1907, p. 6.]

Der englische Kantforscher Green erklärt den Schematismus für eine überflüssige Distinktion ("a surplusage of distinction"). Auch Cäird erklärt den Schematismus für überflüssig, und Adickes bemerkt in seiner Ausgabe der Kritik der reinen Vernunft: „das dunkelste Stück der Kritik haben wir hier vor uns, von manchen deshalb für das tiefsinnigste gehalten. Verschiedenartige Lösungen des Rätsels sind versucht, oft äusserst verwickelte. Ich biete eine neue, sehr einfache, die freilich den Kantgläubigen sehr gewagt, wenn nicht sogar gottlos oder frivol dünken wird. Nach meiner Ansicht ist dem Abschnitt über den Schematismus gar kein wissenschaftlicher Wert beizumessen, da er nur aus systematischen Gründen später in den ,kurzen Abriss' eingefügt ist.“ (Die.

1) Werke (Reclam) 1, 574. 
Das Schematismuskapitel in der Kritik der reinen Vernunft. 339

Äusserungen von Green, Caird und Adickes zitiert H. H. Williams in 'The Monist' 4, $375 \mathrm{ff}$.) Alle diese Schwierigkeiten sollen gelöst werden durch den zweiten Teil der obengenannten Untersuchung von Levy. Diese „soll zeigen, dass jedenfalls eine Auffassung möglich ist, die in dem kritischen Gebäude Kants dem Schematismus nicht die Rolle eines besonderen Ornaments nach Christian Wolff'schem Geschmack, sondern die Bedeutung eines sehr interessanten tragkräftigen architektonischen Gliedes zuweist, welches seine Wirkungen nach allen Richtungen erstreckt." Leider enthält der bisher erschienene erste Teil der Levy'schen Arbeit diese Aufklärungen noch nicht, sondern sie stehen erst im zweiten Teile zu erwarten.

Die Frage nach der systematischen Bedeutung des Schematisnus soll in der folgenden Untersuchung nicht gestellt, eine Entscheidung über Wert oder Unwert des Schemabegriffs nicht herbeigeführt werden. Es soll nur - durch philologische Analyse des Schematismuskapitels - der historische Tatbestand ermittelt werden: was hat Kant unter Schema und Schematismus verstanden wissen wollen?

Der Dunkelheit des Gegenstandes ist es wohl zuzuschreiben, dass die Spezialliteratur über das Schematismuskapitel sehr wenig umfangreich ist. Es gibt eine Dissertation über "Die Kant'sche' Lehre vom Schematismus der reinen Verstandesbegriffe", von P. S. Neide, Halle 1878. Aber man ist nach ihrer Lektüre genau so klug wie zuvor, da sie im Wesentlichen nur eine Paraphrase zum Kantischen Text ist. Aus dem Jahre 1894 stammt ein Aufsatz von H. H. Williams: „Kant's doctrine of the schemata“, in der amerikanischen Zeitschrift "The Monist", 4, 375. Anch von diesem Aufsatz kann nicht gesagt werden, dass er das Verständnis des Schematismuskapitels erleichterte. Die Erklärung des Schemas als "type of experience" ist originell, aber gewiss nicht Kantisch. Unvergleichlich viel wertvoller ist die Arbeit von Walter Zschokke im XII. Band der Kantstudien (1907). Ich habe an einer Stelle meiner Untersuchung ein wichtiges Resultat von Zschokke übernommen (s. u.). Aber im ganzen ist Zschokkes Absicht weniger auf Kantinterpretation als auf systematische Förderung des Problems gerichtet. Endlich besitzen wir in der oben erwähnten Schrift von Levy die letzte Behandlung des Themas. Von dieser Schrift ist aber wie gesagt bisher nur der erste Teil erschienen, und in dem ist vom Schematismus noch gar 
nicht die Rede, sondern nur von den ihm vorangehenden Teilen der Kritik der reinen Vernunft. Daher können wir Levys Buch für unsere Zwecke nicht benutzen: . Soviel als Vorbemerkung. ${ }^{1}$ )

Welches sind die Gedankenreihen in der Kritik der reinen Vernunft, die zur Aufstellung der Lehre vom Schematismus führen? Fassen wir die in der Einleitung, der Ästhetik und der Analytik der Begriffe vorgetragenen Gedanken, soweit sie den Schematismus vorbereiten, kurz zusammen!

Alle menschliche Erkenntnis besteht aus Urteilen. Alle Urteile aber sind entweder analytisch oder synthetisch. Analytisch heissen Urteile, deren Prädikat bloss ein Merkmal des Subjektsbegriffs ist, welches in diesem Subjektsbegriff schon mitgedacht wird. Synthetische Urteile aber sind solche, deren Prädikat zum Subjektsbegriff etwas neues hinzufügt. Es erhellt, dass für den Zuwachs der Erkenntnis nur die synthetischen Urteile Bedeutung haben. Nun ist alle Erkenntnis entweder empirisch, d. h. erzeugt durch die Aktion des Erkenntnisvermögens auf sinnliche Eindrücke, oder apriorisch, d. h. unabhängig von allen sinnlichen Eindrücken "entsprungen". Halten wir diese Bestimmungen mit der Definition der synthetischen Urteile zusammen, so ergibt sich folgendes: diejenigen , synthetischen Urteile, welche empirische Erkenntnis konstituieren („synthetische Urteile a posteriori"), entnehmen die neuen Merkmale, die sie zum Subjektsbegriff hinzufügen, dem unermesslichen Gebiet der sinnlichen Eindrücke. Woher stammen aber die Prädikate in synthetischen Urteilen a priori? Wie sind synthetische Urteile a priori möglich? Dass sie möglich sind, kann nicht in Frage gestellt werden. Denn in der Mathematik, der reinen Naturwissenschaft, und, wenigstens vorgeblich und dem Zwecke nach, in der Metaphysik liegen solche Urteile vor. Die Frage: wie sind synthetische Urteile a priori möglich? zerlegt sich also in folgende Fragen: wie ist Mathematik möglich? Wie ist reine Naturwissenschaft möglich? Wie ist Metaphysik möglich? Die Beantwortung dieser Fragen fällt einer besonderen Wissenschaft zu; der Kritik des apriorischen Erkenntnisvermögens; oder Kritik der reinen Vernunft. Sie heisst auch transzendentale Kritik, wobei transzendental = die Möglichkeit apriorischer Erkenntnis betreffend ist. Die transzendentale Kritik sucht nun die

1) Die vorliegende Arbeit wurde 1908 abgeschlossen. Etwa seither erschienene Spezialliteratur ist nicht eingearbeitet worden. 
Frage nach der Möglichkeit synthetischer Urteile a priori auf die Weise zu lösen, dass sie unsere Erkenntnisvermögen einzeln auf apriorische Elemente hin untersucht. Sie zerfällt demnach - entsprechend den beiden Faktoren oder "Stämmen" der Erkenntnis (Sinnlichkeit und Verstand) in eine transzendentale Sinnenlehre oder Ästhetik und eine transzendentale Verstandeslehre oder Logik. Die transzendentale Logik zerfällt ihrerseits in drei Teile, entsprechend den „drei oberen Erkenntnisvermögen": Verstand (im engeren Sinn), Urteilskraft und Vernunft. (B. - d. i. Kritik der reinen Vernunft, 2. Auflage, 1787 - p. 169.) Dem Verstand und der Urteilskraft entsprechen die beiden Bücher der transzendentalen Analytik, der Vernunft entspricht die transzendentale Dialektik. Das ist der architektonische Grundriss der Kritik der reinen Vernunft. Was ist ihr Inhalt?

Die transzendentale Ästhetik kommt zu dem Ergebnis: alles was uns unmittelbar dụrch die Sinne („anschaulich“) gegeben ist, erleben wir in zwei apriorischen Anschauungsformen, nämlich in Raum und Zeit. Auch der transzendentalen Logik gelingt es apriorische Formen aufzufinden. Es sind die 12 reinen Verstandesbegriffe oder Kategorien. Diese 12 Kategorien sind nurModifikationen einer Verstandeshandlung: der Bearbeitung des Chaos der sinnlichen Eindrücke durch den Verstand („Synthesis des Mannigfaltigen der Anschauung"). Ermöglicht wird diese Synthesis durch die Einheit des Bewnsstseins im Denken, d. i. durch die transzendentale (oder: synthetische) Einheit der Apperzeption. Erst diese macht aus dem Chaos von Empfindungen (Sinneseindrücken) eine Welt von Dingen. Anders ansgedrückt: erst durch die Anwendung der Kategorien anf die sinnliche Anschanung werden Gegenstände erzengt und wird Erkenntnis ermöglicht. Aber die Kategorien sind rein intellektuell, die Anschauungen sind rein sinnlich. Wie ist da die Anwendung der Kategorien auf die Anschaungen möglich?

Die Lösung dieses Problems bietet das Kapitel „vom Schematismus der reinen Verstandesbegriffe“. Dort heisst es: „in allen Subsumtionen eines Gegenstandes unter einen Begriff muss die Vorstellung des ersteren mit dem letzteren gleichartig sein ... . Nun sind aber reine Verstandesbegriffe in Vergleichung mit empirischen (ja überhaupt sinnlichen) Anschauungen ganz ungleichartig und können niemals in irgend einer Anschauung augetroffen werden. Wie ist nun die Subsuntion der letzteren unter die ersten, 
mithin die Anwendung der Kategorie auf Erscheinungen möglich?“ (B. 176). "Nun ist klar, dass es ein Drittes geben müsse, was einerseits mit den Kategorien, andererseits mit der Erscheinung in Gleichartigkeit stehen muss, und die Anwendung der ersteren auf die letzte möglich macht. Diese vermittelnde Vorstellung muss rein (ohne alles empirische) und doch einerseits intellektuell, andererseits. sinnlich sein. Eine solche ist das transzendentale Schema" (ib. 177).

Was ist nun der kantische Schematismus? Es gibt nur eine Art, auf die wir diese Frage beantworten können: wir müssen Kants Äusserungen über Schema und Schematismus der Reihe nach betrachten, $d$. h., wir müssen den Gedankengang des Schematismuskapitels wiedergeben und den Sinn der einzelnen Stellen interpretieren.

Ehe wir jedoch genauer auf die im Schematismuskapitel vorgetragene Lösung des Problems der Anwendung der Kategorien eingehen, werden wir. gut tun, eine Lösung desselben Problems zu betrachten, die Kant an einer anderen Stelle gegeben hat. Denn es darf erwartet werden, dass auf die Schematismuslehre helleres Licht fällt, wenn mạn sie mit einer anderen Behandlung desselben Problems konfrontiert. Diese andere Behandlung findet man in der transzendentalen Deduktion der Kategorien. Dort figuriert nämlich ein $\S 24$, der „von der Anwendung der Kategorien auf Gegenstände der Sinne überhaupt" handelt. Und diese Anwendung der Kategorien auf Gegenstände ist ja gerade das Problem, welches durch den Schematismus gelöst werden soll. Man kann Anstoss nehmen an dem Ausdruck „Gegenstände der Sinne“, weil Gegenstände erst durch die Anwendung der Kategorien auf die Data der Sinnlichkeit erzeugt werden. Aber Kant meint hier mit Gegenständen der Sinne eben solche Data, er meint "das Mannigfaltige in einer sinnlichen Anschauung Gegebene" (B 143). In diesem Paragraphen 24 heisst es nun: „die reinen Verstandesbegriffe beziehen sich durch den blossen Verstand auf Gegenstände der Anschauung überhaupt, unbestinmt, ob sie die unsrige, oder irgend eine andere, doch sinnliche, sei, sind aber eben darum blosse Gedankenformen, wodurch noch kein bestimmter Gegenstand erkannt wird. Die Synthesis oder Verbindung des Mannigfaltigen in demselben bezog sich bloss auf die Einheit der Apperzeption und war dadurch der Grund der Möglichkeit der Erkenntnis a priori, sofern sie auf dem Verstande beruht,- und mithin nicht allein trans- 
Das Schematismuskapitel in der Kritik der reinen Vernunft. 343

zendental, sondern auch rein intellektual. Weil in uns aber eine gewisse Form der sinnlichen Anschauung a priori zum Grunde liegt, welche auf der Rezeptivität der Vorstellungsfähigkeit (Sinnlichkeit) beruht, so kann der Verstand als Spontaneität den inneren Sinn durch das Mannigfaltige gegebener Vorstellungen der synthetischen Einheit der Apperzeption gemäss bestimmen und so synthetische Einheit der Apperzeption des Mannigfaltigen der sinnlichen Anschauung a priori denken, als die Bedingung, unter welcher alle Gegenstände unserer (der menschlichen) Anschauung notwendigerweise stehen müssen; dadurch denn die Kategorien als blosse Gedankenformen objektive Realität, d. i. Anwendung auf Gegenstände, die uns in der Anschaung gegeben werden können, aber nur als Erscheinungen, bekommen, denn nur von diesen sind wir der Anschauung a priori fähig. Diese Synthesis des Mannigfaltigen der sinnlichen Anschauung, die a priori möglich und notwendig ist, kann figürlich (synthesis speciosa) genannt werden" (B 150-151). Die synthesis speciosa wird genauer bestimmt durch die Benennung „transzendentale Synthesis der Einbildungskraft". "Einbildungskraft ist das Vermögen, einen Gegenstand auch ohne dessen Gegenwart in der Anschauung vorzustellen. Da nun alle unsere Anschauung sinnlich ist, so gehört die Einbildungskraft der subjektiven Bedingung wegen, unter der sie allein den Verstandesbegriffen eine korrespondierende Anschauung geben kann, zur Sinnlichkeit; sofern aber doch ihre Synthesis eine Ausübung der Spontaneität ist, welche bestimmend und nicht, wie der Sinn, bloss bestimmbar ist, mithin a priori den Sinn seiner Form nach der Einheit der Apperzeption gemäss bestimmen kann, so ist die Einbildungskraft sofern ein Vermögen, die Sinnlichkeit a priori za bestimmen, und ihre Synthesis der Anschauungen, den Kategorien gemäss, muss die transzendentale Synthesis der Einbildungskraft sein, welches eine Wirkung des Verstandes auf die Sinnlichkeit und die erste Anwendung desselben (zugleich der Grund aller übrigen) auf Gegenstände der uns möglichen Anschaung ist" (B 151-152).

Ich musste Kants eigene Worte anführen, um zu zeigen, dass es sich hier wirklich um dasselbe Problem handelt: um die Anwendung der Kategorien auf die Sinnesdata. Nach § 24 vollzieht sich diese Anwendung in Form einer Synthesis. Ausgeführt wird diese Synthesis von der Einbildungskraft, einem "Grundvermögen der menschlichen Seele" vermittelst dessen "beide äusserste Enden, 
nämlich Sinnlichkeit und Verstand" zusammenhängen (A - d. i. Kritik der reinen Vernunft erste Auflage - 124). Objekt der Synthesis sind aber nicht direkt die Sinnesdata, die empirischen Anschaunngen, sondern "die Formen der sinnlichen Anschauung a priori“, d. i. die reinen Anschauungsformen: Raum und Zeit. Vergleichen wir mit dieser Problemlösung die andere, im Schematismuskapitel dargebotene, so zeigt sich: im Schematismuskapitel wird die Anwendung der Kategorien auf Sinnesdata als Subsumtion (nicht als Synthesis) gefasst, und als Vermittler zwischen Sinnesdaten und Kategorien wird ein neuer Begriff, das transzendentale Schema, eingeführt.

Betrachten wir nun die Schematismuslehre mehr im Detail. Wie so oft bei Kant, so ist auch hier die Terminologie lax und inkonsequent, obwohl Kant weiss, dass philosophische Erkenntnis nicht anders als „nach den strengsten Regeln einer schulgerechten Pünktlichkeit ausgemacht werden kann" (Prolegomena, Reclam p.36). Es besteht nämlich im. Schematismuskapitel ein doppelter Sprachgebrauch in Bezug auf dasjenige, worauf die Kategorien anzuwenden sind. Es werden synonym gebraucht: 1 . Subsumtion von „empirischen (ja überhaupt sinnlichen) Anschauungen" unter Kategorien (B 176) und 2. Subsumtionen der Erscheinungen unter die Kategorien (B 178). "Erscheinungen" und "Anschauungen" sind also als gleichbedeutend gebraucht. Konsequent, exakt ist eigentlich nur die Fintgegensetzung von Kategorien und Anschauungen, denn „Erscheinung" ist oft gleichbedeutend mit „Gegenstand", und dieser Ausdruck wäre hier falsch aus demselben Grunde wie im $\S 24$.

Immerhin ist dies von geringerer Bedeutung, dagegen ist es für das Verständnis des Schematismuskapitels von höchster Wíchtigkeit, dass man sich über die Begriffe "Subsumtion" und "Schema" klar werde.

Das Schematismuskapitel beginnt mit einem Absatz über Subsumtion. Er lautet: „in allen Subsumtionen eines Gegenstandes unter einen Begriff muss die Vorstellung des ersteren mit dem letzteren gleichartig sein, d. h., der Begriff muss dasjenige ent-. halten, was in dem darunter zu subsumierenden Gegenstande vorgestellt wird; denn das bedeutet eben der Ausdruck: ein Gegenstand sei unter einem Begriff enthalten. So hat der empirische Begriff eines Tellers mit dem reinen geometrischen eines Zirkels Gleichartigkeit, indem die Rundung, die in dem ersteren gedacht wird, 
sich in dem letzteren anschauen lässt" (B 176). Was bedeutet hier Subsumtion?

In der tradionellen Logik tritt der Begriff Subsumtion in der Lehre vom Urteil auf. Der ältesten und noch gegenwärtig sehr verbreiteten, wenn auch wissenschaftlich längst überholten Auffassung vom Wesen des Urteils zufolge ist nämlich „das bejahende, elementare Urteil gültig, wenn das Subjekt Art zu der Gattung des Prädikats, der Umfang des Subjekts, also unter den Umfang des Prädikats subsumierbar ist. Nach dieser Subsumtionstheorie ... ist das Subjekt eines jeden gültigen Urteils in scholastischen Ausdruck das contentum, das Prädikat das continens; der Sinn der Knpula: das Enthaltensein des Subjekts in dem Umfang, das Inbegriffen-, Eingeschlossen-, Subsumirtsein unter den Umfang des Prädikats. In etwas allgemeinerer Fassung hat Aristoteles diese Annahme als eine keines Beweises bedürftige Voraussetzung in seinen Äusserungen über den Syllogismus ausgesprochen. Für ihn verbietet sich die Einordnung unter die Beziehung von Art und Gattung infolge seiner engen Fassung dieser beiden Bestimmungen der Gegenstände ... Die gleiche Deutung beherrscht die Entwicklung der abendländischen Urteildeutung im wesentlichen bis in unsere Zeit ... Auch Kant hält trotz mancher Ansätze zu anderer Auffassung an ihr fest". (Erdmann, Logische Elementarlehre $\left.{ }^{2}, 343 / 44\right)$. Ich zitiere die Kant-Stelle, auf die Erdmann sich hier bezieht: „In jedem Urteil ist ein Begriff, der für viele gilt und unter diesem vielen auch eine gegebene Vorstellung begreift, welche letztere dann auf den Gegenstand unmittelbar bezogen wird. So bezieht sich z. B. in dem Urteil: alle Körper sind teilbar, der Begriff des Teilbaren auf verschiedene andere Begriffe; unter diesen aber wird er hier besonders auf den Begriff des Körpers bezogen ... Alle Urteile sind demnach Funktionen der Einheit unter unseren Vorstellungen, da nämlich statt einer unmittelbaren Vorstellung eine höhere, die diese und mehrere unter sich begreift, zur Erkenntnis des Gegenstandes gebraucht und viele ungleiche Erkenntnisse dadurch in eine zusammengezogen werden.“ (B 93, 94.)

Wenn man diese Stelle mit den Bemerkungen über Subsumtion im Schematismuskapitel vergleicht, ergibt sich, dass an beiden Stellen dasselbe gemeint ist. Die Subsumtion eines Gegenstandes unter einen Begriff ist nichts weiter als die Unterordnung eines besonderen unter ein allgemeines im Urteil. 
Nun ist die Urteilstheorie, welche alle Urteile als Subsumtionsakte deutet, völlig unhaltbar. Denn es „bleibt der Subsumtionstheorie, soll sie konsequent sein, nur die Annahme übrig, dass die Beziehung des Teils zum ganzèn, des engeren zum weiteren, die sie behauptet, mit dem . . . Verhältnis der Art zur Gattung zusammenfällt. Denn sie soll eine Beziehung des Umfangs sein. Eine Umfangsvergleichung zweier Gegenstände ist . . . nur unter der Voraussetzung möglich, dass beide als Glieder derselben Ordnungsreihe in Anspruch genommen werden können, sich also wie Art und Gattung verhalten. Diese Konsequenz widerstreitet jedoch dem Urteilsbestande der unzählbaren Fälle, in denen die Gegenstände des Subjekts und des Prädikats verschiedenen Ordnungsreihen zugehören" (Erdmann p. 346). Das Urteil „Die Körper sind teilbar" kann nur dann als Subsumtion aufgefasst werden, wenn man es umformt in: die Körper sind teilbare Dinge. Es ist nämlich möglich (wenn auch gekünstelt) eine Ordnungsreihe zu bilden, in der "Körper" und "teilbare Dinge" auf verschiedenen Stufen vorkommen. Diese Bestimmung nun, dass nur solche Gegenstände in einem Subsumtionsurteil verknüpft werden können, die derselben Ordnungsreihe angehören, kennt auch Kant. Er drückt sie in dem Satze aus: „In allen Subsumtionen eines Gegenstandes unter einen Begriff muss die Vorstellung des ersteren mit dem letzteren gleichartig sein, d. i. der Begriff muss dasjenige enthalten, was in dem darunter $\mathrm{zu}$ subsumierenden Gegenstande vorgestellt wird; denn das bedeutet eben der Ausdruck, ein Gegenstand sei unter einem Begriffe enthalten“. In dieser etwas gewundenen Erklärung kommt aber die Bedingung jeder Subsumtion, nämlich die Zugehörigkeit zur. gleichen Ordnungsreihe, nicht präzise zum Ausdruck, was sich dennn auch rächt: Gleichartig können nämlich auch Begriffe sein, die nicht derselben Ordnungsreihe zugehören, aber ein Merkmal gemein haben. Diese Bedeutung von gleichartig stiehlt sich in den nächsten Satz ein. „So hat der empirische Begriff eines Tellers mit dem reinen geometrischen eines Zirkels Gleichartigkeit, indem die Rundung, die in dem ersteren gedacht wird, sich im letzteren anschauen lässt.“ Gleichartig sind diese-Begriffe allerdings insofern sie das Merkmal der Rundung gemein haben, aber es ist schlechterdings unmöglich, sie in eine Orduungsreihe $\mathrm{zu}$ bringen. Deshalb ist es auch schlechterdings unmöglich sie in einem Subsumtionsurteil zu verknüpfen. Ich kann nicht sagen "Der Teller ist ein Zirkel“. Dann aber ist dieses Beispiel ein völliger Miss- 
griff. Denn es sollte doch das Subsumtionsverhältnis erläutern. Und gerade dazu taugt es nicht... Wie konnte Kant ein solcher Missgriff passieren? Er fasste die Bedingung aller Subsumtion nicht präzis genug, nannte sie mit laxer Formulierung "Gleichartigkeit", ohne zu bemerken, dass Gleichartigkeit eine viel weitere Bedeutung habe. Das Tellerbeispiel bereitet bei der ersten und zweiten Lektüre des Schematismuskapitels viel Kopfzerbrechen, weil man nicht einsieht, inwiefern es zur Illustrierung des Subsumtionsverhältnisses geeignet ist. Man wird, glaube ich, das Beispiel seiner Autorität entkleiden und es einfach unberücksichtigt lassen dürfen. Will man dennoch daran festhalten und das Verhältnis von Kategorie und Schema als Abklatsch dessen zwischen Zirkel und Teller auffassen, so verrennt man sich in eine Sackgasse.

Wir wissen jetzt also, was Kant in unserem Kapitel unter Subsumtion versteht, und können zum nächsten Passus weitergehen, wo die Subsumtion auf einen konkreten Fall angewendet wird, nämlich auf das Verhältnis von Kategorien und Anschauungen oder Erscheinungen. „Nun sind aber, heisst es da, reine Verstandesbegriffe in Vergleichung mit empirischen (ja überhaupt sinnlichen) Anschauungen ganz ungleichartig und können niemals in irgend einer Anschauung angetroffen werden. Wie ist nun die Subsumtion der letzteren unter die ersten, mithin die Anwendung der Kategorie auf Erscheinungen möglich?" Man beachte den. Übergang." Der Gedankengang ist der: Soeben habe ich die Bedingung festgestellt, unter der die Subsumtion eines Gegenstandes unter einen Begriff möglich ist. Nun wird mir zugemutet, die Subsumtion von Erscheinungen unter Kategorien zu vollziehen. Bei dieser mir zugemuteten Subsumtion fehlt aber jene Bedingung. Wie kann ich diese Subsumtion nun doch vollziehen? Die natürliche Antwort hierauf wäre: die Subsumtion ist eben nicht möglich! Wenn Gleichartigkeit der Begriffe zur Subsumtion unerlässlich ist, und die Begriffe sind nicht gleichartig, dann muss man eben auf die Subsumtion verzichten. Hieraus würde nun weiter folgen, dass die Anwendung der Kategorien auf die Erscheinungen, wenn anders sie möglich sein soll, eine andere Form haben muss als die der Subsumtion. Kant zieht diesen Schluss nicht. Er hilft sich weiter auf eine etwas sophistische Art, und man muss ihm scharf auf die Finger sehen. Er sagt: „Nun ist klar, dass es ein drittes geben müsse, was einerseits mit der Kategorie, andererseits mit der Erscheinung in Gleichartigkeit stehen 
muss und die Anwendung der ersteren auf die letztere möglich macht". Diese Behauptung scheint dem Leser durchaus nicht so selbstrerständlich, wie Kant sich schmeichelt. Aber dieses „nun ist klar" hat bei Kant dieselbe Bedeutung wie bei Spinoza das "nt per se notum": beide werden zuweilen als Dekorationsstücke aufgeklebt, um einen Riss in der Mauer zu verdecken. Item, hören wir weiter: „Diese vermittelnde Vorstellung muss rein (ohne alles empirische) und dcch einerseits intellektuell, andererseits sinnlich sein. Eine solche ist das transzendentale Schema" (B 177). Als transzendentales Schema entpuppt sich die "transzendentale Zeitbestimmung“. „Daher wird eine Anwendung der Kategorien auf Erscheinungen möglich sein vermittelst der transzendentalen Zeitbestimmung, welche als das Schema der Verstandesbegriffe die Subsumtion der letzteren unter die erste vermittelt" (B 178). Diese Auskunft ist wirklich überraschend! Vorhin hatten wir klipp und klar erkannt: eine Subsumtion der Erscheinungen unter die Kategorien ist ausgeschlossen - und jetzt wird uns eben diese Subsumtion wieder als vollziehbar und vollzogen angepriesen. Man steht scheinbar vor einem völligen Widersinn. Scheinbar! Denn: die hier proponierte Subsumtion ist eben etwas ganz anderes als die, von der früher die Rede war. Stillschweigend und unbemerkt hat Kant einen anderen Subsumtionsbegriff eingeschmuggelt. Um ihn zu verstehen, müssen wir Kants logische Lehren berücksichtigen. Subsumtion ist ein Terminus in Kants Lehre vom Schluss. In der Kantischen Logik wird in $\S 57$ das allgemeine Prinzip aller Vernunftsschlüsse folgendermassen formuliert: „Was unter der Bedingung seiner Regel steht, das steht auch unter der Regel selbst". Dazu die Anmerkung: „Der Vernunftschluss prämittiert eine allgemeine Regel und eine Subsumtion unter die Bedingung derselben". Ebenda lautet der $\S 58$ „Wesentliche Bestandstücke des Vernunftschlusses": "Zu einem jeden Vernunftschlusse gehören folgende wesentliche drei Stïcke: 1 . eine allgemeine Regel, welche der Obersatz (propositio major) genannt wird, 2. der Satz, der eine Erkenntnis unter die Bedingung der allgemeinen Regel subsumirt und der Untersatz (propositio minor) heisst, und endlich 3. der Satz, welcher das Prädikat der Regel von der subsumirten Erkenntnis bejaht oder verneint, der Schlussatz (conclusio). Die beiden ersteren Sätze werden in ihrer Verbindung mit einander die Vordersätze oder Prämissen genannt". Dazu die Anmerkung: „Eine Regel ist eine Assertion unter einer allgemeinen Bedingung ... 
Die Erkenntnis, dass die Bedingung (irgendwo) stattfinde, ist die Subsumtion". Hierzu vergleiche man noch B 386: „Vernunft als Vermögen einer gewissen logischen Form der Erkenntnis betrachtet, ist das Vermögen zu schliessen, d. i. mittelbar (durch die Subsumtion der Bedingung eines möglichen Urteils unter die Bedingung eines gegebenen) zu urteilen. Das gegebene Urteil ist die allgemeine Regel (Obersatz, major). Die Subsumtion der Bedingung eines anderen möglichen Urteils unter die Bedingung der Regel ist der Untersatz (minor). Das wirkliche Urteil, welches die Assertion der Regel in dem subsumirten Falle aussagt, ist der Schlusssatz (conclusio)." 1)

Dieser Subsumtionsbegriff gehört also der Lehre vom Schluss an, während der zuerst behandelte der Urteilslehre angehört. Die Verschiedenheit der beiden Subsumtionsbegriffe bedarf keines Beweises. Dieser bezog sich auf die Unterordnung eines Gegenstandes unter einen Begriff, jener bezieht sich auf die Unterordnung eines Falles unter eine Regel. Nur mit letzteren kommen wir in der Schemalehre aus. Das Schema ist ja, wie wir später sehen werden, eine Regel. Jede Kategorie hat ihr Schema, d. h. ihre transzendentale Zeitbestimmung. So ist Beharrlichkeit in der Zeit das Schema der Substantialität.

Wo ich also Beharrlichkeit in der Zeit wahrnehme, da habe ich die Kategorie der Substantialität anzuwenden, ein Verhältnis, das sich so ausdrücken lässt: Wo A gilt, gilt B. Wo das Schema gilt, gilt seine Kategorie, d. h., wo ich an einer Erscheinung den eigentümlichen Rythmus wahrnehme, der als transzendentale Zeitbestimmung das Schema einer bestimmten Kategorie ist, da gilt diese Kategorie für jene Erscheinung, da muss die Kategorie auf die Erscheinung angewandt werden. Die Beziehung, welche durch das Schema zwischen Kategorie und Erscheinung gestiftet wird, hat also die Form eines Schlusses von der Gestalt:

1) Ditse Terminologie konnte Kant bei Wolf finden. In dessen ${ }_{n}$ Philosophia rationalis sive Logica methodo scientifica pertractata et ad usum scientiarum atque vitae aptata" (Francofurti et Lipsiae 1728) heisst es ( $(362)$ : Propositio minor sub maiore dicitur subsumi, si haec primo ponitur loco. E. gr. siquis affirmaverit: Nullus hominum in omnibus est sapiens, et alter responderit: Atqui tu es homo, hanc propositionem subsumere dicitur. Sumta vero majore ex concesso et subsumta minore propter evidentiam; sponte deinde sua sequitar conclusio: Ergo tu in omnibus non es sapiens. 


\section{Wenn A gilt, so gilt $X$}

(cf. Sigwart, Logik ${ }^{8}$ 1,434).

A gilt bei $B$

also $X$ gilt bei $B$

In diesem Schluss heisst nun nach Wolfisch-Kantischer Terminologie der Untersatz „Subsumtion “. In unserem Kapitel aber überträgt Kant diesen Namen voni Untersatz auf die Konklusion (die ja im Untersatz schon drin steckt), und macht sich dadurch einer kleinen Inkonsequenz gegen seine eigene Terminologie schuldig. Wenn man sich alles dies klar gemacht hat, ist man befähigt zum Verständnis des Satzes: „Daher wird eine Anwendung der Kategorie auf Erscheinungen möglich sein, vermittelst der transzendentalen Zeitbestimmung, welche als das Schema der Verstandesbegriffe die Subsumtion der letzteren unter die erste vermittelt".

Hiermit schliesse ich meine Bemühungen zur Deutung des Begriffs „Subsumtion“ ab. Als Resultat ergibt sich: Das Wort Subsumtion hat in Schematismuskapitel zwei verschiedene Bedeutungen, worüber Kant den Leser im Unklaren lässt.

Wir können jetzt weitergehen zur Erörterung des Begriffs Schema. Zunächst wird das Schema bestimmt als ein drittes, "was einerseits mit der Kategorie, andererseits mit der Erscheinung in Gleichartigkeit stehen muss". Diese Bestimmung folgt notwendig aus dem Begriff der Subsumtion. Das Schema ist ein tertium zwischen reinen Verstandesbegriffen und Anschauungen und muss daher „rein (ohne alles Empirische) und doch einerseits intellektuell, andererseits sinnlich sein". Zunächst kann man sich unter einem solchen Gebilde garnichts denken. Es gibt nur einerseits Anschauungen (Sinnlichkeit), andererseits Kategorien (Verstand). Und das sind sogen. "disjunkt-koordinierte Begriffe". Tertium non datur. Hier scheint eine durch den Subsumtionsbegriff herbeigeführte unüberwindliche Schwierigkeit vorzuliegen. Aber sehen wir weiter. Vielleicht löst sie sich durch die weitere Ausführung. Da stellt sich nun heraus, dass in Gestalt der transzendentalen Zeitbestimmung ein Wesen vorhanden ist, welches alle Qualifikationen zur Uebernahme der von einem transzendentalen Schema geforderten Dienste. besitzt. Transzendentales Schema wird also die transzendentale Zeitbestimmung. Die Sache ist noch nicht klar, und man erwartet, Kant werde nun dazu übergehen, die verschiedenen Arten der transzendentalen Zeitbestimmung, welche als transzendentale Schemata den verschiedenen Kategerien entsprechen, aufzuzählen. 
Aber es kommt ganz anders. Diese Spezifikation der transzendentalen Schemata erfolgt erst fünf Seiten später (p. $182 \mathrm{ff}$.). Und was liegt dazwischen? Eine neue, zweite Ableitung des Schemas! Diese auffallende Erscheinung, zu welcher der immer neu ansetzende Beweisgang in der transzendentalen Deduktion der Kategorien eine Parallele bildet, wird zwar nicht hinreichend erklärt, verliert aber viel von ihrem überraschenden Charakter durch folgende Bemerkung von Vaihinger:

„Die Publikationen der "Losen Blätter“, der „Reflexionen“ und des "Opus postumum" aus Karts Nachlass haben uns über die Arbeitsmethode Kants belehrt: wir finden überall einzelne kürzere oder längere Ausführungen, wobei Kant in immer nur neuen Ansätzen den spröden Gegenstand zu bewältigen sucht, und bei diesen neuen Ansätzen nimmt Kant auf seine eigene früheren Darstellungen fast nie Rücksicht. Er setzt fast immer wieder neu ein, ohne Beziehung auf die schon vorliegenden älteren Aufzeichnungen. Dadurch erklären sich sowohl die immer neuen Behandlungen desselben Themas als die auffallenden Abweichungen derselben voneinander". (Vaihinger, die transzendentale Deduktion der Kategorien; 1902, p. 1).

Sehen wir nan die neue Ableitung des Schemas an. Der Anfang schliesst sich an das Vorausgehende in keiner Weise an: „Nach demjenigen, was in der Deduktion der Kategorien gezeigt worden, wird hoffentlich niemand in Zweifel stehen, sich über die Frage zu entschliessen: ob diese reinen Verstandesbegriffe von bloss empirischem oder auch von transzendentalem Gebrauche seien, d. h. ob sie lediglich als Bedingungen einer möglichen Erfabrung sich a priori auf die Erscheinungen beziehen, oder ob sie als Bedingungen der Iröglichkeit der Dinge überhaupt auf Gegenstände an sich selbst (ohne einige Restriktion auf unsere Sinnlichkeit) erstreckt werden können. Denn da haben wir gesehen, dass Begriffe ganz unmöglich sind, noch irgend einige Bedeutung haben können, wo nicht entweder ihnen selbst, oder wenigstens den Elementen, daraus sie bestehen, ein Gegenstand gegeben ist, mithin auf Dinge an sich (ohne Rücksicht, ob und wie sie uns gegeben werden mögen) gar nicht gehen können, dass ferner die einzige Art, wie ein Gegenstand gegeben werde, die Modifikation unserer Sinnlichkeit sei; endlich, dass reine Begriffe a priori ausser der Funktion des Verstandes in der Kategorie noch formale Bedingungen der Sinnlichkeit (namentlich des inneren Sinnes) a priori enthalten 
müssen, welche die allgeneine Bedingung enthalten, unter der die Kategorie allein auf irgend einen Gegenstand angewandt werden kann. Wir wollen diese formale und reine Bedingung der Sinnlichkeit, auf welche der Verstandesbegriff in seinem Gebrauch restringiert ist, das Schema dieses Verstandesbegriffs und das Verfahren des Verstandes mit diesen Schemata den Schematismus des reinen Verstandes nennen." (178-179.) Bei dieser Deduktion des Schemas fällt uns vor allem auf, dass sie ganz ohne Hilfe des Begriffs Subsumtion zustande kommt. Zweitens fällt uns auf, dass sie auf etwas basiert "Was in der Deduktion der Kategorien gezeigt worden".

Dieses Fundament der Deduktion ist in dem oben besprochenen § 24 zu erblicken, denn das ist der Ort, wo bewiesen worden war, „dass reine Begriffe a priori ausser der Funktion des Verstandes in der Kategorie noch formale Bedingangen der Sinnlichkeit (namentlich des inneren Sinnes) a priori enthalten müssen." Dort waren die formalen Bedingungen unter dem Namen der Synthesis speciosa oder der transzendentalen. Synthesis der Einbildungskraft zusammengefasst worden. Dort war eben, wie oben gezeigt, dasselbe Problem wie im Schematismuskapitel behandelt worden. Und während das Schematismuskapitel in seiner Lösung des Problems bisher ganz andere IVege gegangen war als der § 24, wird jetzt, in dieser zweiten Ableitung des Schemas, der Faden des früheren Gedankenganges (in $\S 24$ ) wieder aufgenonımen. Die Beziehung zwischen den beiden Gedankenreihen (Schema einerseits, synthesis speciosa andererseits) wird deutlicher in dem folgenden Satz: „Das Schema ist an sich selbst jederzeit nur ein Produkt der Einbildungskraft, aber indem die Synthesis der letzteren keine einzelne Anschauung, sondern die Einheit in der Bestimmung der Sinnlichkeit allein zur Absicht hat, 'so ist das Schema doch vom Bilde zu unterscheiden". Hier kehrt also der Begriff "Synthesis der Einbildungskraft" wörtlich wieder, mit der wichtigen Bestimmung, die Schemata der Verstandesbegriffe seien Produkte dieser Synthesis. ${ }^{1}$ ) . Diese Bestimmung klärt das Verhältnis zwischen dem Schema und der transzendentalen Synthesis der Einbildungskraft, und damit das Verhältnis zwischen dem § 24 und dem Schematis-

1) Die Beziehung zwischen Schema und Einbildungskraft, welche hier in dem Urteil ausgedrückt ist „das Schema ist ein Produkt der Einbildungskraft", wird auf p. 180 durch das Genitivverhaltnis "Schema der Einbildungskraft" ausgedrückt. 
muskapitel völlig auf. Das Problem der Anwendung der Kategorien auf die Erscheinungen wird schon in dem $\$ 24$ aufgestellt und gelöst. Die Problemlösung, die in der zweiten Ableitung des Schemas im Schematisnuskapitel geboten wird, ist nichts weiter als eine Wiederholung und genauere Ausführung der in $\$ 24$ vorgetragenen Lösung. Die in $\S 24$ eingeführte Mittelinstanz zwischen Kategorie und Erscheinung wird im Schematismuskapitel in Beziehung auf die einzelnen Kategorien spezifiziert. Aus der einen transzendentalen Synthesis der Einbildungskraft werden zwölf transzendentale Schemata, gerade wie seiner Zeit aus der einen intellektualen Synthesis zwölf Kategorien wurden. Dass die transzendentalen Schemata Zeitbestimmungen sind, das konnte man auch schon aus $\S 24$ wissen, denn dort war gesagt, dass es der "innere Sinn", die Form der sinnlichen Anschauung a priori, mithin die Zeit sei, welche durch die transzendentale Synthesis der Einbildungskraft bestimmt werde. Will man noch einen Beweis für die enge Beziehung, welche zwischen dem Schematismuskapitel und dem $\S 24$ besteht, so findet man einen solchen in einer handschriftlichen Eintragung Kants in seinem Handexemplar der Kritik der reinen Vernunft. Er fügt dort, nach Erdmanns Bericht, „zu dem Wort Schematismus die Überschrift: ,Die Synthesis des Verstandes, wenn sie den inneren Sinn der Einheit der Apperzeption gemäss bestimmt, heisst so'. Damit, sagt der Herausgeber Erdmann, ist eine für ihren Zusammenhang wichtige nähere Bestimmung der transzendentalen Schemate ausgesprochen, die zwar der Sache nach, aber nicht in dieser präzisen Formulierung von Kant gegeben ist" (Nachträge zu Kants Kritik der reinen Vernunft, aus Kants Nachlass herausgegeben von B. Erdmann, Kiel 1881, pp. 27-28). Es ist bemerkenswert, dass auch in dieser nachträglichen Definition des Schematismus der Gedanke der Subsumtion ganz fallen gelassen ist. Jedenfalls bedarf es keines Beweises, dass die beiden Auffassungen des Schematismus (d. h. der Anwendung der Kategorien), diejenige als Subsumtion und die als Synthesis, einander schroff entgegengesetzt sind. Die Schwierigkeit und Dunkelheit des Schematismuskapitels aber beruht nicht zum wenigsten auf der Einführung des Begriffs Subsumtion. Bisher ist Schema immer

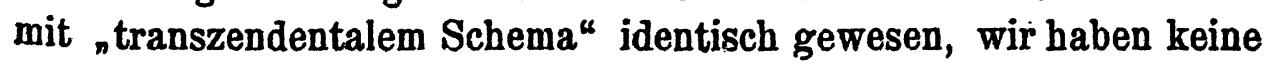
andern Schemata gekannt als die transzendentalen, und diese waren uns aus transzendentalen Erwägungen erwachsen, nicht aus der psychologischen Empirie übernommen. Diese transzendentale Ab- 
leitung des transzendentalen Schemas ist der Grund, weshalb man in Übereinstimmung mit Kuntze (Die kritische Lehre von der Objektivität, 1906, p. 145) alle psychologistischen Deutungen des Schematismus als verfehlt bezeichnen darf. Aber der nächste Absatz ergänzt und bereichert unser Wissen vom Schema. Wir werden erstens darüber aufgeklärt, dass das transzendentale Schema nur eine Airt der Gattung Schema ist, und zweítens wird uns der ausgedehnte Gebrauch der Schemata als Tatsache der empirischen Erkenntnispsychologie an einigen Beispielen dargelegt, d. h. der transzendentalen Deduktion des Schemas wird eine metaphysische zur Seite gestellt. Hierdurch präzisiert sich unsere Vorstellung von dem, was wir uns unter einem Schema zu denken haben. Zunächst werden wir darauf aufmerksam gemacht, dass das Schema nicht mit einem Bilde verwechselt werden darf. „So, wenn ich fünf Punkte hintereinander setze ......, ist dieses ein Bild von der Zahl fünf. Dagegen, wenn ich eine Zahl überhaupt nưr denke, die nun fünf oder hundert sein kann, so ist dieses Denken mehr die Vorstellung einer Methode; einem gewissen Begriffe gemäss eine Menge (z. E. tausend) in einem Bilde vorzustellen, als dieses Bild selbst, welches ich im letzteren Falle schwerlich würde übersehen, und mit dem Begriff vergleichen können." Eine Erläuterung zu dieser Stelle bietet Schelling. „Das Schema“, sagt er in seinem ,System des transzendentalen Idealismus", „nuss unterschieden werden sowohl vom Bild als vom Symbol ... Das Bild ist immer von allen Seiten so bestimmt, dass zur völligen Identität des Bildes mit dem Gegenstand nur der bestimmte Teil des Raumes fehlt, in welchem der letztere sich befindet. Das Schema dagegen ist nicht eine von allen Seiten bestimmte Vorstellung, sondern nur Anschauung einer Regel, nach welcher ein bestimmter Gegenstand hervorgebracht werden kann“. (Sämtliche Werke, 1: Abt., 3,508.) Nachdem der Irrtum, das Schema sei ein Bild, abgewiesen ist, kann Kant eine präzise Definition des Begriffes geben: „Die Vorstellung nun von einem allgemeinen Verfahren der Einbildungskraft, einem Begriff sein Bild zu verschaffen, nenne ich das Schema zu diesem Begriffe“. Diese Definition können wir zwar intellektuell apprehendieren, aber. wir sind noch ausser Stande, eine lebendige Vorstellung mit dem, was darin intendiert ist, zu verbinden. Darum führt Kant den "Gängelwagen der Urteilskraft", d. h. Beispiele (B 174 oben), ein. Vom Standpunkt einer Phänomenologie des Bewusstseins aus geht Kant dazu über, Schemata und Schematismus als tatsächlich vor- 
handen und funktionierend aufzuzeigen. Bedauerlicherweise leiden auch diese Ausführungen Kants an einer Dunkelheit, welche nicht aus Tiefsinn, sondern aus unklarer und hilfloser Ausdrucksweise entspringt. Das Schema ist die Vorstellung von einem Verfalıren, einem Begriff sein Bild zu verschaffen. Der Begriff hat also im Verhältnis zum Schema logische Priorität und das Schema steht in der Mitte zwischen Begriff und Bild. Nun will Kant beweisen, dass überall da, wo einem Begriff soin Bild verschafft werden soll, dies durch das Schema des Begriffes vermittelt wird. Zuerst beweist er dies bei reinen sinnlichen Begriffen. Solche Begriffe sind die Begriffe von geometrischen Figuren. Man erwartet nun, Kant wird sagen: unsern Bildern von reinen sinnlichen Begriffen liegen nicht diese Begriffe, sondern deren Schemata zu Grunde. Statt dessen sagt Kant: „In der Tat liegen unsern reinen sinnlichen Begriffen nicht Bilder der Gegenstände, sondern Schemate zum - Grunde ${ }^{\text {. }}$. Diese Überleitung mit „In der Tat" erinnert an das oben charakterisierte ${ }_{n}$ nun ist klar". Beide Ausdrücke bedeuten, dass der logische Faden abgerissen ist, und sollen einen Sprung in der Darstellung verbergen. So steht es -denn auch mit dem eben zitierten Satze. Hier ist das Verhältnis: Begriff $\rightarrow$ Schema $\rightarrow$ Bild ganz verschoben, und es sieht so aus, als hätten die Schemata logische Priorität im Verhältnis zu den Begriffen. Das muss man wenigstens aus dem Ausdruck „zum Grunde liegen“ folgern. Das widerspricht aber der Definition des Schemas. Das Schema ist ja gerade durch seine Abhängigkeit von einem Begriff gekennzeichnet und definiert. Daran lässt sich nicht rütteln. Die Schwierigkeit scheint unlösbar. Sie lässt sich jedoch vielleicht lösen, wenn wir den Wortsinn des „zu Grunde liegen“, nicht in seiner Starrheit bestehen lassen. $\mathrm{Zu}$ dieser freieren Auslegung fühlen wir uns dadurch autorisiert, dass Kant später an entsprechender Stelle einen andern Ausdruck wählt, wie wir weiter unten sehen werden. Es handelt sich um das Verhältnis Begriff $\rightarrow$ Schema $\rightarrow$ Bild. Das hat er in dem Anstoss erregenden Satze einfach umgedreht und gesagt:

$$
\text { Bild } \rightarrow \text { Schemá } \rightarrow \text { Begriff. }{ }^{1} \text { ) }
$$

Diese Umkehrung kann man natürlich vornehmen. Sie verhält sich zu der andern Formel wie Erkenntnisgrund zu Realgrund. Sie tangiert also jene Formel in ihrer transzendentalen Wahrheit nicht. Immerhin bleibt es dabei, dass dieses Triangelbeispiel verwirrend Begriffe.

1) Genauer: Bilder der Gegenstände $\rightarrow$ Schemata $\rightarrow$ reine sinnliche 
wirkt, weil es die Gedankenentwicklung nicht gradlinig fortfïhrt. Eine Abirrung bedeutet es auch, wenn auf die Inadäquatheit des Triangelbildes im Verhältnis zum Triangelbegriff solches Gewicht gelegt wird. Diese Inadäquatheit ist Privateigentümlichkeit des Triangels, gehört aber keineswegs zum Wesen des Verhältnisses von Begriff $\rightarrow$ Schema $\rightarrow$ Bild. Dies sieht man leicht an einem andern "reinen sinnlichen Begriff“, z. B. dem des Kreises oder des Quadrats. Hier ist von einer solchen Inadäquatheit zwischen Bild und Begriff keine Rede. Eben darum aber durfte Kant auch beim Triangel das Existieren und Funktionieren des Schemas nicht auf diese Inadäquatheit stützen.

Auf diesen Passus, den man, wenn es sich nicht um Kant, sondern um einen handschriftlich überlieferten antiken Autor handelte, wegen innerer Unstimmigkeiten vielleicht als Interpolation ausmerzen würde, folgt die Bestimmung, das Schema des Triangels bedeute eine Regel der Synthesis der Einbildungskraft in Ansehung reiner Gestalten im Raume. Damit sind wir wieder in dem uns vertrauten Gedankengang drin.

Hatten die soeben besprochénen Ausführungen Kants den reinen sinnlichen Begriffen gegolten, so werden sie jetzt durch entsprechende Bestimmungen über ,empirische Begriffe ${ }^{\natural}$ ergänzt. „Noch viel weniger, hören wir, erreicht ein Gegenstand der Erfahrung oder Bild desselben jemals den empirischen Begriff, sondern dieser bezieht sich jederzeit unmittelbar auf das Schema der Einbildungskraft". Dieser Satz korrespondiert dem oben besprochenen: „In der Tat liegen unsern reinen sinnlichen Begriffen nicht Bilder der Gegenstände, sondern Schemate zum Grunde“. Nur sind die Ausdrücke anders gewählt, dieselben Relationen abweichend formuliert. Das ist sehr interessant, für das Verständnis wertvoll. Das Verhältnis der beiden Sätze stellt sich so dar:

1. Bilder der Gegenstände $\rightarrow$ Schemate $\rightarrow$ reine sinnliche Begriffe.

2. Gegenstand d. Erf. oder Bild desselben $\rightarrow$ Schema $\rightarrow$ empirischer Begriff.

Der Unterschied zwischen 1. und 2. liegt in der Bedeutung. der Pfeile. In 1 . bedeuten sie "zum Grunde liegen" - und an diesem Ausdruck hatten wir Anstoss genommen. In 2. bedeuten die Pfeile „bezieht sich". Hier ist also der irreleitende Ausdruck „zum Grunde liegen“ ersetzt durch den neutralen Ausdruck „sich beziehen". So hat sich Kant selbst korrigirt. Der Ausdruck: der 
Das Schematismuskapitel in der Kritik der reinen Vernunft. 357

Begriff beziehe sich auf das Schema, nicht auf das Bild, steht mit der logischen Priorität des Begriffes im Verhältnis zum Schema nicht im Widerspruch wie es der Ausdruck tut: dem Begriff liege das Schema zum Grunde.

Das Verhältnis von Begriff und Schema wird aber ganz klar durch die neue Definition des Schemas, die Kant nun gibt: Das Schema ist eine Regel der Bestimmung uuserer Anschauung gemäss einem gewissen allgemeinen Begriffe. Diese zweite Definition ist viel klarer und brauchbarer als die erste. Das Schema ist nicht mehr „die Vorstellung von einem allgemeinen Verfahren", sondern "eine Regel". Und zwar nicht „eine Regel, einem Begriffe sein Bild zu verschaffen“, sondern „eine Regel der Bestimmung unserer Anschauung gemäss einem Begriffe“. Nach dieser Definition lässt sich nicht mehr daran zweifeln, dass der Begriff die logische Priorität vor dem Schema hat. Man wird jene Stelle, aus der sich die umgekehrte Auffassung ergab, als irrefübrend bezeichnen müssen. Das Schema wird gebildet gemäss einem Begriff, es ist eine Projektion des Begriffs auf die Anschauung. Wollen wir das Verhältnis ganz deutlich machen, so können wir mit einem mathemathischen Ausdruck sagen: Jedes Schema ist Funktion eines Begriffs. Dieser Punkt ist sehr wichtig. Dass das Schema Funktion eines Begriffes ist, dies ${ }_{\beth}$ ist das wesentliche Merkmal des Schemas. In diesem Zusammenhang darf darauf hingewiesen werden, dass diese funktionelle Abhängigkeit des Schemas von seinem Begriff in der Subsumtionstheorie garnicht zum Ausdruck kommt. Im Gegenteil: unsere Erklärung der Subsumtion von Erscheinungen unter Kategorien vermittelnder Schemata verdrehte dieses Verhältnis zwischen Begriff und Schema völlig. Wir hatten die Subsumtion dargestellt mit Anlehnung an die Schlussformel

Wo A gilt, gilt $\mathrm{X}$

$\begin{array}{r}A \text { gilt bei } B \\ \hline \text { also } X \text { gilt bei } B\end{array}$

wobei $A$ das Schema, $X$ die Kategorie, $B$ die Erscheinung bedeutete. In dieser Formel kommt aber der Charakter des $\mathrm{A}$ als Funktion von $\mathrm{X}$ nicht nur nicht zum Ausdruck, sondern es wird eher der Eindruck erweckt, als ob $\mathrm{X}$ sich in einer Abhängigkeit von A befinde. Nun ist aber, nach meinem Erachten, die in dieser Formel niedergelegte Deutung des Subsumtions-Schematismus die einzig mögliche. In dieser Überzeugung bestärkt mich Falckenbergs Deutung des Schematismus. Falckenberg deutet näm- 
lich den Subsumtions-Schematismus auf dieselbe Weise (wenn anch in anderem Ausdruck) wie ich, und vom Synthesis-Schematismus hört man bei ihm nichts. Er sagt zum Beispiel: Das Dasein zu einer bestimmten Zeit sei der Fingerzeig für die Kategorie der Wirklichkeit (Falckenberg, Hilfsbuch zur Geschichte der Philosophie seit Kant ${ }^{2}$, p. 17). Dieser Satz drückt genau das aus was ich in jener Schlussformel ausgedrückt hatte, und zeigt deutlich, dass die Auffassung des Schematismus als Subsumtion inadäquat ist, weil sie das Wesen des Schemas, Funktion eines Begriffes zu sein, verdeckt, anstatt es auszudrücken. Nach diesem Exkurs über Subsumtions-Schematismus können wir Kants Ausführungen über das Schema wieder Gehör leihen. Zuvor empfiehlt es sich jedoch, Schellings Erklärung des Schemas wieder mit der von Kant zu vergleichen. Kant hatte das Schema eine Regel der Bestimmung unserer Anschauung genannt. Vielleicht kommt diese Charakteristik des Schemas zu noch schärferer Ausprägung in Schellings Worten: „Das Schema ist Anschauung der Regel, nach welcher ein bestimmter Gegenstand hervorgebracht werden kann". (Sämtliche Werke, 1. Abt. 3, 508.) "Das Schema zeigt sich im gemeinsten Verstandesgebrauch als das allgemeine Mittelglied der Anerkennung jedes Gegenstandes als eines bestimmten" (ib. p. 509). „Das Schema ist die sinnlich-angeschaute Regel der Hervorbringung eines empirischen Gegenstandes" (ib. p. 510). Halten wir dies fest: das Schema ist eine Regel! Kant erläutert nun seine Schematheorie noch an einem Beispiel. „Der Begriff vom Hunde bedeutet eine Regel, nach welcher meine Einbildungskraft die Gestalt eines vierfüssigen Tieres allgemein verzeichnen kann, ohne auf irgend eine einzige besondere Gestalt, die mir die Erfahrung dazu bietet, oder auch ein jedes mögliche Bild, was ich in concreto darstellen kann, eingeschränkt zu sein". Dieser Satz enthält eine neue Überraschung: "Der Begriff rom Hunde bedeutet eine Regel". Und da Regel = Schema, können wir sagen: Der Begriff vom Hunde bedeutet ein Schema. Hier fliesst also der Begriff eines Dinges mit seinem Schema zusammen! Diese sehr interessante Peripetie steht zwar im Widerspruch zu dem ganzen vorhergehenden Passus, wo zwischen Begriff eines Dinges und Schema desselben immer scharf geschieden wurde, harmoniert dafür aber umsomehr mit unserer eigenen psychologischen Erfahrung. Es ist uns kaum möglich, aus unserem inneren Erleben heraus die Koexistenz von Schema und Begriff eines Hundes, oder eines Triangels, als 
distinkter Faktoren nachzufühlen. Das was Kant Schema nennt deckt sich mit dem was wir uns unter Begriff denken! In diesem Sinne sagt Riehl: „Um die Allgemeinheit des Begriffes mit der Individualität der Anschauung auszugleichen, bedarf es nach Kant einer Klasse von Zwischengebilden, die die Natur der begrifflichen Vorstellungen mit der der anschaulichen teilen: eben der Schemata. Irre ich nicht, so sind es gerade diese Schemata selbst, welche allein im eigentlichen Sinne Begriffe sind und was darüber hinausliegt, ist nur noch das Wort das die Vorstellungen bezeichnet, aber keine für sich irgend fassbare Vorstellung mehr". (Der philosophische Kritizismus I ${ }^{2}, 533$.) Indessen: äiese Auffassung, wonach Begriff und Schema ein und dasselbe sind, wird bei Kant nur durch die eine Stelle (Hundebeispiel) gestützt, läuft aber im übrigen der ganzen Schematismuslehre stracks zuwider. Sie wird dadurch begünstigt, dass es uns in praxi schwer fällt, auf Grund unserer inneren Erfahrung Begriff und Schema eines Dinges zu unterscheiden. Und doch muss dem aus transzendentalen Gründen eingeführten Begriff des Schemas eine psychologische Realität entsprechen - sonst stürzt das transzendentale Gebäude zusammen. Deshalb hat ja Kant der transzendentalen Deduktion des Schemas eine "metaphysische“ zur Seite gestellt. Wie die reinen Anschauungsformen und die reinen Verstandesbegriffe werden die Schemata nicht nur in ihrer transzendentalen Bedeutung für die Möglichkeit der Erkenntnis, sondern auch als empirische Tatsachen der Phänomenologie des Bewusstseins aufgezeigt. So ist auch der Satz zu verstehen: "Dieser Schematismus unseres Verstandes in Ausehung der Erscheinungen ist eine verborgene Kunst in den Tiefen der menschlichen Seele, deren wahre Handgriffe wir der Natur schwerlich jemals abraten und sie unverdeckt vor Augen legen werden". Noch deutlicher drückt sich Schelling aus. Er erklärt den Schematismus für einen Vorgang, „welchen jeder nur aus eigener innerer Erfahrung kennen lernen, und den man, um ihn kenntlich zu machen und die Erfahrung zu leiten, nur beschreiben, und von allem andern, was ibm ähnlich ist, absondern kann". (S. W. 1. Abt., 3, 505.) Trotz dieser psychologischen Einsprengungen aber ist an der transzendentalen Grundtendenz des Schematismuskapitels festzuhalten. Ob das Schema als psychologische Realität aus dem Trümmerhaufen der Kantischen Psychologie zu retten ist, und ob die moderne Psychologie etwas damit anzufangen 
weiss, das zu untersuchen wäre zwar sehr interessant, gehört jedoch nicht hierher.

Kant muss nun, nachdem er vom Schemata im allgemeinen gesprochen hat, den Weg zurückfinden zum transzendentalen Schema. Er tut dies in folgenden Sätzen: "Das Bild ist ein Produkt des empirischen Vermögens der produktiven Einbildungskraft, das Schema sinnlicher Begriffe (als der Figuren im Raume) ein Produkt und gleichsam ein Monogramm der reinen Einbildungskraft a priori, wodurch und wonach die Bilder allererst möglich werden, die aber mit dem Begriffe nur immer vermittelst des Schemas, welches sie bezeichnen, verknüpft werden müssen und an sich demselben nicht völlig kongruieren. Dagegen ist das Schema eines reinen Verstandesbegriffs etwas, was in gar kein Bild gebracht werden kann, sondern ist nur die reine Synthesis gemäss einer Regel der Einheit nach Begriffen überhaupt, die die Kategorie ausdrückt, und ist ein transzendentales Produkt der Einbildungskraft, welches die Bestimmungen des inneren Sinnes überhaupt, nach Bedingungen seiner Form (der Zeit) in Ansehung aller Vorstellungen betrifft, sofern diese der Einheit der Apperzeption gemäss a priori in einem Begriff zusammenhängen sollen." (181.) Also das Schema einer Kategorie ist "die reine Synthesis gemäss einer Regel der Einheit nach Begriffen überhaupt, die die Kategorien ausdrückt". Hier bezieht sich das Relativum die (quam) doch wohl auf Regel. "Regel der Einheit nach Begriffen" wäre dann also eine Umschreibung für Kategorie, und wir könnten lesen: „das Schema einer Kategorie ist die reine Synthesis gemäss dieser Kategorie". Also: das transzendentale Schema ist eine Modifikation der Synthesis, keine Subsumtion. Aber mit dieser Bestimmung ist der Begriff des transzendentalen Schemas noch nicht erschöpft. Er ist ein transzendentales Produkt der Einbildungskraft - das Schema überhaupt war ,jederzeit nur ein Produkt der Einbildungskraft" gewesen - und es bestimmt den inneren Sinn, wie das Schema überhaupt "eine Bestimmung unserer Anschauung“. ist, und zwar bestimmt es den inneren Sinn nach Bedingungen seiner Form (der Zeit) in Ansehung aller Vorstellangen. Das transzendentale Schema ist also eine von der Einbildungskraft ausgeübte Synthesis der Vorstellungen in der Zeit (als Form des inneren Sinnes), gemäss einer Kategorie. Vergleichen wir wieder die Formulierung Schellings: „Das empirische Schema wurde erklärt als die sinnlich angeschaute Regel, wonach ein Gegenstand empi- 
risch hervorgebracht werden kann. Das transzendentale wird also die sinnliche Anschauung der Regel sein, nach welcher ein Objekt überhaupt, oder transzendental hervorgebracht werden kann" (p.516). Die Definition des transzendentalen Schemas, zu der wir jetzt gelangt sind, schliesst sich wieder aufs engste an die Ausführungen in $\S 24$ (B 150 unten) an. Wir können jetzt einen Schritt weitergehen und die einzelnen transzendentalen Schemata spezifizieren. „Die Schemate sind daher nichts als Zeitbestimmungen a priori nach Regeln, und diese gehen nach der Ordnung der Kategorien auf die Zeitreihe, den Zeitinhalt, die Zeitordnung, endlich den Zeitinbegriff in Ansehung aller möglichen Gegensiände." (p. 184.)

Kant macht nun die einzelnen Schemata namhaft. Wir brauchen hierauf nicht im Detail einzugehen, da für die prinzipiellen Fragen, die sich aus dem Schematismuskapitel ergeben, nichts dabei herauskommt. Ein kurzer Überblick über die Ausgestaltung der Schematheorie wird genügen. Kant hat bekanntlich- zwölf Kategorien. Er musste also auch zwölf transzendentale Schemata aufzählen können. Das gelingt ihm aber nicht, er bringt nur 9 zu Stande. Das Schema der Quantität ist die Zahl. (Die Quantität ist aber keine von den 12 Kategorien, sondern der gemeinsame Titel für die drei ersten.) Das Schema der Realität ist das Sein in der Zeit, das der Negation das Nichtsein in der Zeit. Das Schema der Substanz ist die Beharrlichkeit des Realen in der Zeit, das der Kausalität „die Succession des Mannigfaltigen, insofern sie

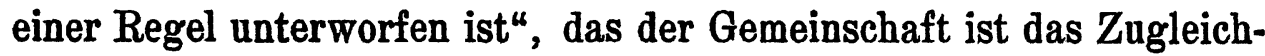
sein der Bestimmungen einer Substanz mit denen einer andern. Schema der Möglichkeit ist die Zusammenstimmung der Synthesis verschiedener Vorstellungen mit den Bedingungen der Zeit überhaupt, das der Wirklichkeit ist das Dasein in einer bestimmten Zeit, das der Notwendigkeit endlich das Dasein eines Gegenstandes zu aller Zeit. Über diese Tafel der Schemata urteilt Zschokke: „Es ist ganz unmöglich, das auszuführen, was Kant unterliess, and wenn wir die einzelnen Schemata genau nachprüfen, die angegeben sind, so geraten wir in ein Nebelmeer von Unklarheiten hinein ". (Kantstndien XII, 169.) Demselben Autor entnehme ich folgende kurze, aber einschneidende Kritik der Schemata, die durch ihre vorzügliche Formulierung zur wörtlichen Anführung zwingt: „Das Schema sollte nach Kant ein Drittes sein zwischen Anschaunng und Verstandesbegriff, und worin besteht es tatsächlich? Es ist nichts mehr und nichts weniger als die 
Verbindung von Anschauung und Begriff selber, die doch eben das Problem war: die Zeit ist die Anschauungsform, die Kategorie ist der Verstandesbegriff, das Schema ist eine Vereinigung beider, sonst nichts; anstatt eines Dritten, welches wir suchten, legt Kant Eins und Zwei kurzerhand zusammen. Das Problem wird dadurch höchst einfach gelöst, dass es ignoriert wird. So heterogen Anschauung und Begriffe sein mögen, wie Kant zunächst behauptete, im Schema verbindet er sie durch den Machtspruch: fügt euch zusammen. Das dritte zur Anwendung wird Kant unter den Händen die Anwendung selber.“ (Kantstudien XII, 169.) Zschokke kommt zu dem Schluss, „dass Kants Begriff des Schemas an keiner Stelle wirklich klar erkennen lässt, dass es ein Tertium zwischen Begriff und Anschauung sei“ (ib. 170/171). Diese Kritik bestätigt die Bedenken, die ich gleich anfangs erhoben hatte, als ich an dem Begriff des Tertium zwischen Begriff und Anschauung Anstoss nahm.

Fassen wir nun die Ergebnisse unserer bisherigen Untersuchungen zusammen. Wir waren zu folgendem Resultat gelangt: Das Schematismusproblem ist das Problem der Anwendung der Kategorien auf Anschauungen. Dieses Problem entwickelt sich organisch aus der Analytik der Begriffe. Nachdem das Erkenntnisvermögen zerfasert worden ist in reine Anschauungsformen und in reine Verstandestormen, muss sich die Frage erheben: „Wie beziehen sich die reinen Verstandesformen auf die Anschauungen?" Diese Frage hat Kant gegen Ende der Analytik, in $\S 24$, beantwortet. Die Antwort lautet: „Die Anwendung der Kategorien auf »Gegenstände der Sinne * wird vermittelt durch ihre Anwendung auf.die reinen Anschauungsformen Raum und Zeit. Diese Anwendung vollzieht sich in Form einer apriorischen Synthesis, welche die Kategorien auf diese reinen Anschauungen ausüben. Diese Synthesis heisst synthesis speciosa. Da sie durch die Einbildungskraft vollzogen wird, heisst sie auch transzendentale Synthesis der Einbildungskraft". Diese Gedanken werden nun im Schematismuskapitel wieder aufgenommen und dahin ergänzt, dass die Formen, in welchen sich diese Synthesis der Einbildungskraft vollzieht, als transzendentale Schemata bezeichnet und spezifiziert werden. Die Schematismuslehre ist also nur eine organische Weiterbildung und Ausgestaltung der Kategorienlehre. Deshalb meint auch Rięhl, sie hätte „ebensogut, ja natürlicher im Zusammenhang der Deduktion der Kategorien behandelt werden können“. (Der philosophische 
Das Schematismuskapitel in der Kritik der reinen Vernunft. 363

Kritizismus, $\left.1^{2}, 532\right)$. Neben dieser Gedankenreihe finden wir im Schematismuskapitel eine andere Ableitung der Schemata, die von dem $\S 24$ ganz unabhängig ist, die Anwendung der Kategorien auf die Anschauungen als Subsumtion der letzteren unter die ersteren auffasst und aus dieser Subsumtion den Begriff des Schemas herleitet. Es erhebt sich nun die Frage: wie verhalten sich die beiden Auffassungen des Schematismus zu einander? Wie verhält sich der Subsumtionschematismus zum Synthesis-Schematismus? Und da sich die beiden Auffassungen widersprechen, stellt sich die Frage so: welche Auffassung verdient den Vorzug? Aus unserer ganzen Darlegung ergibt sich, dass alle Chancen zu Gunsten des Synthesisschematismus, zu Ungunsten des Subsumtionsschematismus sprechen. Denn

1. Der Subsumtionsschematismus drängt sich unorganisch in die vom § 24 zum Synthesisschematismus gradlinig verlaufende Gedankenreihe hinein.

2. Der Subsumtionsschematismus spielt im weiteren Verlauf des Schematismuskapitels gar keine Rolle mehr.

3. Die Ausführungen über Subsumtion leiden an inneren Widersprüchen.

4. Der Subsumtionsschematismus bringt das Wesen des Schemas nicht zum Ausdruck.

5. Subsumtion ist ein logischer, Synthesis ein erkenntnistheoretischer Begriff. Das Wesen des Schematismus ist aber erkenntnistheoretisch, wird also durch den Ausdruck Synthesis adäquater bezeichnet als durch den Ausdruck Subsumtion. Aus diesen Gründen verdient der Synthesisschematismus entschieden den Vorzug.

Man fragt sich nur: Wie ist Kant dazu gekommen, diesen gekünstelten und seine Aufgabe so mangelhaft erfüllenden Subsumtiousschematismus einzuführen, wo er doch in dem - schon in $\S 24$ angedeuteten - Synthesis-Schematismus eine viel befriedigendere und organischere Lösung des Problems bereit hatte? Die Einführung des Subsumtionsschematismus liegt meines Erachtens. nicht in dem Problem selbst begründet, sondern - in dem Zwang, den die von Kant gewählte Systematik auf die schriftstellerische Darstellung seiner Gedanken ausübte. Man vergegenwärtige sich die Stellung des Schematismuskapitels in dem architektonischen Grundriss der Kritik der reinen Vernunft. Das Kapitel steht am Anfang des zweiten Buches der transzendentalen Analytik, der 
Analytik der Grundsätze. Nun ist aber, wie wir zu Anfang dieses Buches erfahren, „die allgemeine Logik“ „über einem Grundriss erbaut, der ganz genau mit der Einteilung des oberen Erkenntnisvermögen zusammentrifft. Diese sind Verstand, Urteilskraft und Vernunft. Jene Doktrin handelt daher in ihrer Analytik von Begriffen, Urteilen und Schlüssen" (B. 169), wobei also die Begriffe dem Verstand, die Urteile der Urteilskraft, die Schlüsse der Vernunft entsprechen. Im Wesen der transzendentalen Logik ist es nun bedingt, dass sie das Einteilungsprinzip der "allgemeinen Logik“ nur mit der Modifikation übernehmen kann, dass von der Vernunft nicht in der Analytik, sondern in der Dialektik gehandelt und die Analytik zwischen Verstand und Urteilskraft geteilt wird. Nun entsprach das erste Buch der transzendentalen Analytik, die Analytik der Begriffe, dem Verstand. „Die Analytik der Grundsätze (deren erstes Kapitel das Schematismuskapitel ist) wird demnach lediglich ein Kanon für die Urteilskraft sein, der sie lehrt, die Verstandesbegriffe, welche die Bedingung zu Regeln a priori enthalten, auf Erscheinungen anzuwenden" (B 171). Ailso: alle Ausführungen der Analytik der Grundsätze, mithin auch das Schematismuskapitel, stehen unter dem Zeichen der Urteilskraft. Darum heisst die Analytik der Grundsätze auch „transzendentale Doktrin der Urteilskraft" (B 176). Was ist aber denn die Urteilskraft? Darauf erhalten wir die Antwort: „Wenn der Verstand überhaupt als das Vermögen der Regeln erklärt wird, so ist Urteilskraft das Vermögen, unter Regeln zu subsumiren, d. i. zu unterscheiden, ob etwas unter. einer gegebenen Regel (casus datae legis) stehe oder nicht" (B 171).

Die Urteilskraft wird bestimmt als das Vermögen zu subsumiren! Also muss das, was in dem "transzendentalen Doktrin der Urteilskraft" betitelten Abschnitt zur Sprache kommt, in irgend einer Beziehung zu dem Subsumiren stehen! Nun sollte die transzendentale Doktrin der Urteilskraft ${ }_{n}$ zwei Hauptstïcke enthalten: das erste, welches von der sinnlichen Bedingung handelt, unter welcher reine Verstaudesbegriffe allein gebraucht werden können, d. i. von dem Schematismus des reinen Verstandes, das zweite aber von denjenigen synthetischen Urteilen, welche aus reinen Verstandesbegriffen unter diesen Bedingungen a priori herfliessen und allen übrigen Erkenntnissen a priori zum Grunde liegen, d. i. von den Grundsätzen des reinen Verstandes“ (B 175).

Also musste in dem Schematismus von Subsumtion die Rede 
Das Schematismuskapitel in der Kritik der reinen Vernunft. 365

sein. Darum hat Kant, obwohl er den Synthesisschematismus schon bereit hatte, versucht, den Schematismus noch in das Schema einer Subsumtion $\mathrm{zu}$ bringen - und so ist der Subsumtionschematismus entstanden. Merkwürdig bleibt allerdings noch immer, weshalb Kant das Schematismuskapitel mit dem irreführenden Passus über die Subsumtion von Gegenständen unter Begriffe eingeleitet hat, anstatt gleich den Begriff der Subsumtion einzuführen, den wir uns aus der Kant-Jäsche'schen Logik und aus B 386 herausgeklaubt haben, und der auch schon in der oben zitierten Definition der Urteilskraft (B 171) steckt.

Aus dem Gesagten folgt, dass es irreführend ist, wenn wie dies gewöhnlich geschieht - in den Darstellungen des Schematismus nur von Subsumtion die Rede ist. Meines Erachtens hat jede Interpretation und Darstellung des Schematismus von der transzendentalen Synthesis auszugehen - und die Subsumtionstheorie kann als unwesentlich und störend bei Seite gelassen werden.

$\mathrm{Zu}$ bemerken ist noch, dass dem Subsumtions-Schematismus und dem Synthesis-Schematismus die Auffassung des Schemas als transzendentaler Zeitbestimmung gemeinsam ist. In $\S 24$ ist diese Auffassung noch nicht scharf durchgeführt. Zwar wird auch dort gesagt, die figürliche Synthesis bestimme den inneren Sinn - und dessen Form ist ja die Zeit, oder sie bestimme „die sinnliche Anschaung a priori“, d. h. die Zeit, die ja den Raum unter sich befasst. Aber doch wird diese Synthesis figürlich, speciosa genannt, welche Benennung doch offenbar eine Beziehung auf den Raum ausdrückt: allein, es ist zweifellos konsequenter, wenn der Schematismus ein zeitlicher ist, weil die Zeit die übergreifende Anschauungsform ist. Deswegen wird man Ueberweg nicht zustimmen können, wenn er sagt, aus denselben Gründen wie die Zeit scheine auch der Raum einen Schematismus liefern zu können und $\mathbf{z u}$ müssen. (Ueberweg-Heinze, Gesch. d. Philosophie III ${ }^{9}$ p. 328 Anm.)

Die Verwerfung des Subsumtionsschematismus ist nicht ohne Wirkung auf unsere Auffassung vom Wesen des Schemas. Im Verlanf des Schematismuskapitels traten uns zwei Auffassungen vom Wesen des Schemas entgegen: das Schema als Tertium und das Schema als Regel. Die beiden Auffassungen sind unverträglich, inkommensurabel, und die Auffassung als Tertinm war uns von Anfang an verdächtig erschienen. Nun können wir sie mit gutem Gewissen über Bord werfen - denn sie war ja nur bedingt durch den Subsumtionsschematismus. Wenn der Mantel fällt, muss 
der Herzog nach! Bemerkenswert ist auch, dass Schelling, der Kants Schematismuslehre in seinem "System des transzendentalen Idealismus" ganz orthodox wiedergibt, von dem Subsumtionsschematismus ebenso wie von dem Schema als Tertium nichts weiss, und das Schema durchweg als Regel auffasst.

Wenn man davon absieht, an der Lehre vom Schematismus Kritik zu üben, und es nur darauf anlegt, Kants Gedanken wie derzugeben, dann muss man sagen: der Schematismus hat in Kants Erkenntnislehre eine zentrale Bedeutung, denn durch ihn werden "die beiden äussersten Enden“, Verstand und Sinnlichkeit wieder zusammengefügt, nachdem sie völlig isoliert worden waren. Nur der Schematismus macht das menschliche Denken möglich. Unser ganzes geistiges Leben beruht auf ihm. Ohne Schematismus hätten wir nur einerseits ungeformte sinnliche Eindrücke, andererseits unerfüllte erkenntnistheoretische Formen. Der Schematismus ist fest vernietet in Kants erkenntnistheoretischem System. Dies sieht man auch daraus, dass Kants Lehre von der Zeit sich hier „als ein unentbehrliches Zwischenglied seiner gesamten psychologischerkenntnistheoretischen Konstruktion" zeigt. (Windelband, Geschichte der neueren Philosophie, ${ }^{4}, \Pi$, 82.)

Freilich steht und fällt die Schematismuslehre mit einer Voraussetzung: der Diskrepanz und damit der Vermittlungsbedürftigkeit von Verstand und Sinnlichkeit. Wenn man diese Voraussetzung nicht zugibt, dann bräucht man auch den Schematismus nicht zu akzeptieren, wenigstens nicht in der von Kant ihm gegebeuen Form. Das ist die Stellung Zschokkes. Nach ihm ist die Diskrepanz der beiden "Stämme" ein Überbleibsel aus Kants. vorkritischer Periode. Verstand und Sinnlichkeit ergänzen sich, sie bilden nicht einen Gegensatz, den der Schematismus erst überbrïicken müsste. So meint auch Uंeberweg: „Es bedarf nicht eines besonderen Schematismus, da ja schon die Gestaltung des sinnlich gegebenen Stoffes durch die beiden Anschauungsformen überhaupt denselben zu der ferneren Gestaltung durch die Kategorien präpariert". (Ueberweg-Heinze III ${ }^{9}$ p. 328 Anm.) 